\title{
Size effects in visual recognition memory are determined by perceived size
}

\author{
BRUCE MILLIKEN and PIERRE JOLICOEUR \\ University of Waterloo, Waterloo, Ontario, Canada
}

\begin{abstract}
Recognition memory for shapes has been shown to depend on differences between the size of shapes at the time of encoding and at the time of the memory test (Jolicoeur, 1987). Experiment 1 of the present paper replicates this effect and establishes a set of parameters used in the subsequent experiments. Experiment 2 considers the results of Experiment 1 in light of the distinction between "perceived" size, which, under normal viewing conditions, varies minimally with changes in distance between the observer and object, and "retinal" size, which varies proportionally with viewing distance as an object is moved closer to or farther from an observer. Subjects studied novel shapes and performed a recognition memory test in which the distance from the subject to the viewing screen at the time of testing was different from that at the time of encoding. The viewing distance and the size of the shapes were manipulated such that perceived and retinal sizes were dissociated. The results suggest that the size-congruency effect in memory for visual shape occurs as a result of changes in the perceived size of shapes between the encoding and the testing phases, with little or no contribution of retinal size per se.
\end{abstract}

It has long been established that alterations in the orientation of a shape can affect one's ability to recognize that shape (Rock, 1956, 1973, 1974; Rock, Di Vita, \& Barbeito, 1981; Rock \& Heimer, 1957). Such effects have been attributed to the fact that changes in orientation can lead to "changes in perceived shape," thereby provoking failure of the observer to make contact with the appropriate internal representation. Rock $(1974$, p. 81) notes, however, that alterations of size, color, or type of contour do not change perceived shape. In so saying, Rock (1974) implies that internal representations of shape are orientation dependent but are independent of other variables, such as size. If this is the case, then recognition memory should not be affected by alterations in the size of shapes.

However, recent studies by Jolicoeur (1987) and Kolers, Duchnicky, and Sundstroem (1985) have shown that recognition memory is sensitive to size as well. The studies presented in this paper are concerned with the effects of size on recognition memory; the method used to study size effects is very similar to that used by Jolicoeur (1987). Jolicoeur had subjects study a group of shapes that were of two different sizes. Following this study phase, subjects participated in a recognition phase involving the set of shapes that the subjects had studied and an identical number of shapes not seen previously. Of the shapes seen previously, half of the shapes that were studied small were

This research was supported by a postgraduate scholarship from the Natural Sciences and Engineering Research Council awarded to Bruce Milliken, and by grant OGP0000795 from the Natural Sciences and Engineering Research Council awarded to Pierre Jolicoeur. The authors would like to thank Walter Gogel and two anonymous reviewers for their helpful comments on an earlier draft of this paper. Correspondence should be addressed to Bruce Milliken, Department of Psychology, University of Waterloo, Waterloo, Ontario N2L 3G1, Canada. shown small at test time, and half were shown large. Similarly, half of the shapes studied large were shown large at test time, and half were shown small. The subjects' task in the test phase was to decide as quickly and accurately as possible whether or not they had studied the shape before, while ignoring any size differences. Subjects recognized shapes that were studied and tested at the same size more quickly and accurately, in general, than they recognized those studied at one size and tested at a different size. Jolicoeur (1987) labeled this pattern of results a size-congruency effect. The term size congruency will be used in this paper as well and will refer to the relationship between the size of a shape at encoding and the size of the same shape during testing.

Jolicoeur (1987) concluded that size-dependent recognition performance suggests that shapes may be encoded in long-term memory in a format that is size dependent. Granted this possibility, none of the previous studies examining size effects on recognition memory performance have addressed the distinction that can be made between retinal size, which varies with the distance between the external pattern and the observer, and perceived size, which, under normal viewing conditions, remains constant despite said changes in distance. If the distance between the observer and the external pattern is the same during encoding as during the recognition test, then changes in the objective size of a shape results in corresponding changes in both the retinal and perceived size of the shapes. In short, without changes in the distance between the observer and the external pattern, retinal and perceived size are confounded with one another. Therefore, even if the recognition task is sensitive to the format of representations in long-term memory, to conclude that "size" is represented in that format is, de facto, an under- 
description. A question that begs answering is whether it is retinal or perceived size that is encoded as part of the representation. The experiments described in the present paper address this issue by having subjects make recognition decisions from a distance that decouples retinal size from perceived size.

\section{EXPERIMENT 1}

Experiment 1 was designed to replicate the sizecongruency effect observed by Jolicoeur (1987), as well as to establish a set of size parameters that would serve for the remaining experiments. The method used differed from that of Jolicoeur (1987) in a number of respects. The stimuli were adapted from the set of $\mathbf{4 0}$ stick figures used in Experiment $1 \mathrm{~b}$ by Jolicoeur (1987). However, 5 of the 40 stick figures that contained closed portions (i.e., " 4 " has a closed portion, " $F$ " does not) were modified so that none of the $\mathbf{4 0}$ shapes contained closed portions in the present experiments. The reason for this change was that, with so few of the shapes having closed portions, it seemed possible that the closed portion would be used as a memory cue irrespective of the rest of the shape. The shapes were presented as white antialiased lines ${ }^{1}$ (Tanner, Jolicoeur, Cowan, Booth, \& Fishman, 1989) on a black background, instead of the black lines on white background used by Jolicoeur (1987). Shapes were displayed on the monitor of an Amiga 1000 microcomputer rather than on a two-way projection screen or on the monitor of an Apple II microcomputer. Finally, a size ratio of 2:1 was chosen for the "large" and "small" shapes used in this study. None of the changes to the Jolicoeur (1987) procedure was expected to affect the results on its own; however, because there were a large number of small changes, it seemed desirable to replicate the size-congruency effect using the above procedure before attempting to measure potential variations in the effect in further studies.

\section{Method}

Subjects. The subjects were 23 undergraduates and 1 graduate student at the University of Waterloo, who were paid for their participation. All subjects had normal or corrected-to-normal vision. The subjects were tested by five different experimenters, four of whom tested 4 subjects each. The final 8 subjects were tested by one of the authors.

Materials. Each stick figure was made up of six line segments, with the constraints that each segment met another segment only at its endpoint and that, otherwise, line segments could not cross over one another. Each stick figure consisted of one segment of length 10 units, two segments of length 5 units, and three segments of length 3 units, similar to those used by Jolicoeur (1987). The shapes were displayed as white antialiased lines (Tanner at al., 1989) on a black background on an Amiga 1000 microcomputer monitor. The average visual angle subtended by the shapes was $3.2^{\circ}$ when presented at the small size and $6.4^{\circ}$ when presented at the large size. Examples of the shapes may be seen in Figure 1.

Procedure. Each subject participated in one session lasting about $20 \mathrm{~min}$. Approximately half of this time was spent informing the subject of the task before it began and debriefing the subject after the task ended. There were two phases to each session: a study phase and a test phase. During the study phase, the subject was asked to rest his/her chin in a chinrest and to study shapes as they ap-
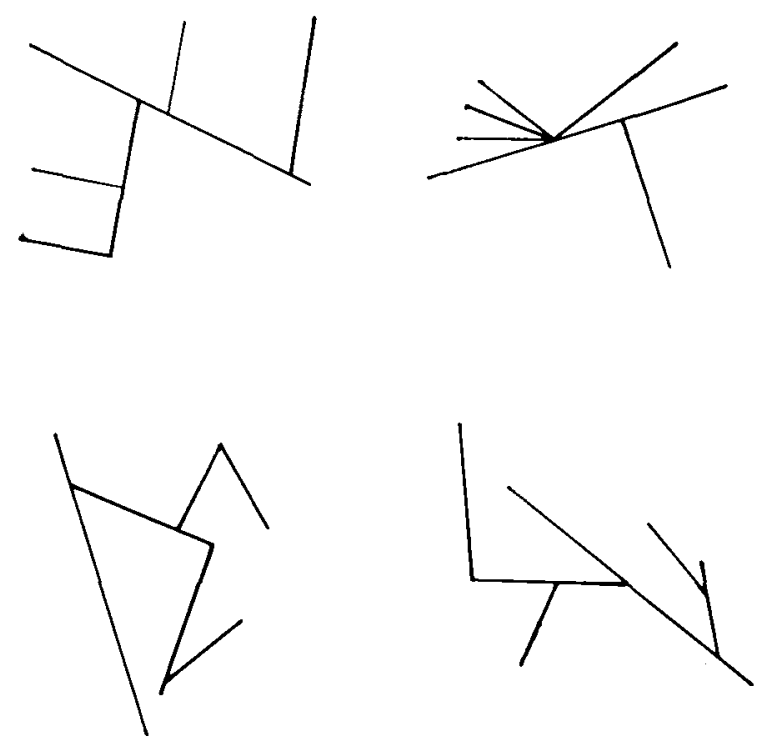

Figure 1. Examples of shapes used in the experiments.

peared one at a time on the monitor, which was located $83 \mathrm{~cm}$ in front of the subject. The subjects were informed before the study phase that their recognition memory for the shapes would be tested immediately after the study phase. They were also informed that size differences between shapes were to be considered irrelevant. Immediately following the study phase, the subjects participated in a test phase in which shapes were again presented to the subject one at a time. The subjects were asked to discriminate, as quickly and accurately as possible, whether or not the shape on the screen was one of the ones they had studied.

All 40 shapes were used in each of the experimental sessions. The shapes were divided at random into two sets of 20 shapes (Set A and Set B). Half of the subjects studied Set A items, and the other half studied Set B items. There were 40 presentations in the study phase, consisting of two presentations of each of the shapes from one of the two sets. There were $\mathbf{4 0}$ presentations in the test phase as well, consisting of one presentation of each of the shapes from both sets. Thus, in the test phase, the subject had seen exactly one half of the shapes before (targets), and exactly one half of the shapes were new (foils). The order of presentation of the shapes within both the study phase and the test phase was random; in the test phase, no more than four targets or four foils were allowed to appear consecutively.

Of the set of 20 shapes that the subject studied, 10 were shown at a small size $\left(3.2^{\circ}\right)$ during the study phase and 10 were shown at a large size $\left(6.4^{\circ}\right)$. In the subsequent test phase, of the 10 shapes studied small, 5 were shown small and 5 were shown large. Similarly, of the 10 shapes studied large, 5 were shown large and 5 were shown small. Thus, at each of the test sizes, half of the shapes were shown at the same size as that when studied and half were shown at a different size. Moreover, the groups of 5 shapes that comprised each of these four cells for a particular subject were broken up, and the shapes were distributed among the remaining three cells for the subsequent subjects. The set of 20 shapes that the subject did not study served as foils in the test phase. Foils were counterbalanced in the same manner as targets, with study size as a dummy variable. In the full design, then, a shape could be studied large or small, testod large or small, and be a target or a foil. Each of the $\mathbf{4 0}$ shapes was represented in each of the possible eight cells of the full design once every 8 subjects.

In the study phase, each of the 20 target shapes was shown once to the subject and then shown again to the subject in a different 
random order, with no break between the two presentations of the set of shapes. Each shape appeared in the middle of the computer monitor for $6 \mathrm{sec}$. The shape then disappeared and was followed by a blank interval of $2 \mathrm{sec}$. The presentation of the next target shape followed the blank interval. In all, the study phase lasted approximately $5 \mathrm{~min}$. The subjects were then asked to start the test phase after being reminded of response-button hand assignments. Each test phase trial consisted of a fixation point for $750 \mathrm{msec}$, then a blank interval for $500 \mathrm{msec}$, followed by the test shape. The test shape stayed on the screen until the subject made a response. The subject rested the index finger of each hand on separate response buttons and was asked to press the response button corresponding to his/her dominant hand if the shape was a target or to press the nondominant-hand response button if the shape was a foil. Response time (RT) was measured as the interval between the onset of the shape and the response of the subject. There was an intertrial interval of $2 \mathrm{sec}$ between the subjects' response and the fixation point of the next trial. The first three trials of the test phase were always foils. This was done so that the errors that are often associated with a subjects' first few buttonpresses would occur on foils, which (as is described below) were of less theoretical interest than the target trials.

The target trials provide a situation where a correct response ostensibly requires a match between the external test shape and an internal representation of that shape. The sensitivity of this process to size mismatches suggests that size is encoded in this internal representation. Moreover, since study size was a dummy variable for the foils in this experiment, the study size $\times$ test size interaction that characterizes a size-congruency effect was not expected for the foils. For this reason, an analysis of the foils is presented after that for the targets and is considered only briefly.

\section{Results}

The mean correct RTs on target trials for each subject at each study and test size were submitted to a repeated measures analysis of variance (ANOVA), treating study size and test size as within-subject factors. ${ }^{2}$ These means collapsed across subjects are shown in Figure 2.

The only significant result was the interaction between study size and test size $\left[F(1,23)=4.52, M S_{\mathrm{e}}=\right.$ $\left.5.031771 \times 10^{s}, p<.05\right]$. It is clear in Figure 2 that whether subjects responded more quickly to shapes tested small or to shapes tested large depended on the size at which the shapes were studied. In other words, faster RTs were associated with shapes that were shown at the same size in both the study phase and the test phase.

Note that the study size $\times$ test size interaction depicted in Figure 2 is not a perfectly symmetric crossover interaction. The mean RT for shapes whose test size was large $(1,770 \mathrm{msec})$ was moderately slower than that for shapes tested small $(1,550 \mathrm{msec})(p<.15)$.

The mean percent error rates on target trials for each subject, study size, and test size were submitted to the same ANOVA as were the RTs (the mean percent error rates for each study size and test size are displayed in the bottom panel of Figure 2). Again, the only significant effect was the study size $\times$ test size interaction $[F(1,23)=$ $\left.10.89, M S_{\mathrm{e}}=1.2391 \times 10^{2}, p<.004\right]$. In agreement with the RTs, there was a tendency to make fewer errors on shapes that were shown at the same size in both the study phase and the test phase.
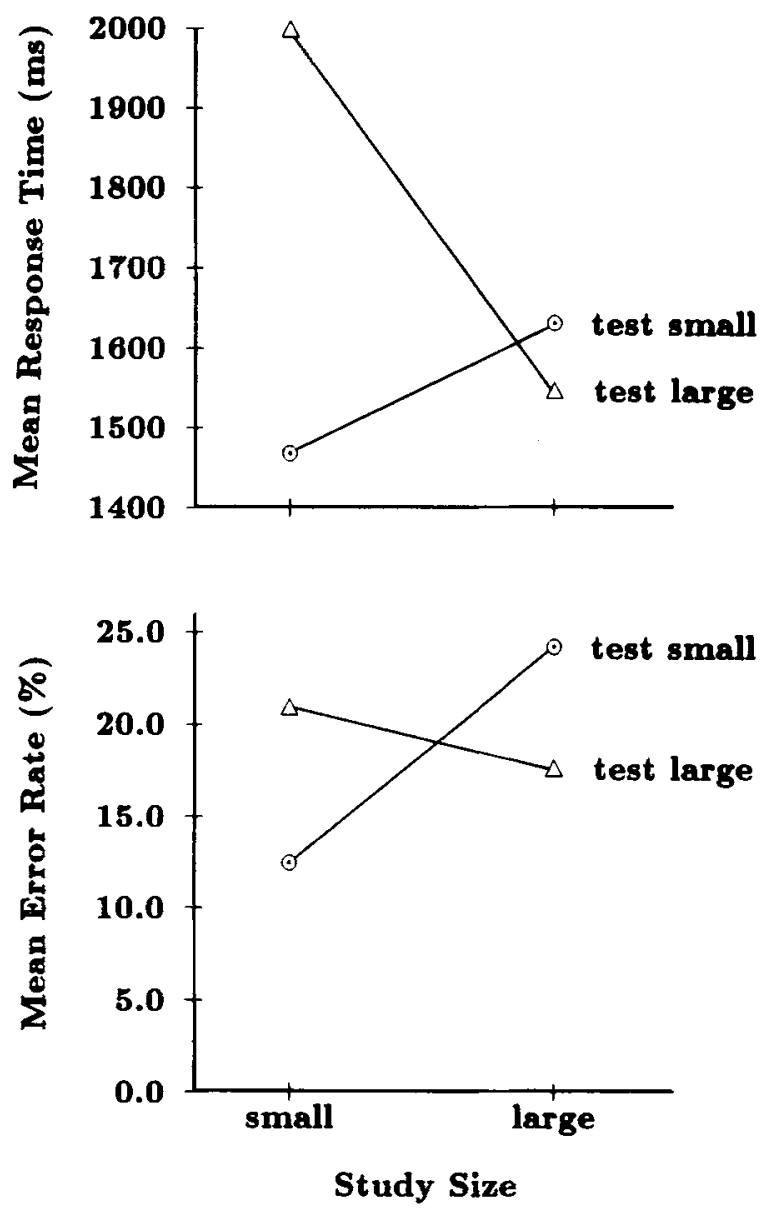

Figure 2. Mean response times and mean percent error rates in Experiment 1.

Analysis of foils. The data gathered from each subject for the foils alone were submitted to a repeated measures ANOVA, with study size and test size as within-subject factors. For both RTs and error rates, there were no significant effects ( $p$ s $>.20$, in all cases).

Also, the data from each subject for both targets and foils were submitted to a repeated measures ANOVA with target/foil, study size, and test size as within-subject factors. Target shapes were responded to significantly faster $(1,660 \mathrm{msec})$ than were foils $(2,524 \mathrm{msec})[F(1,23)=$ $\left.29.27, M S_{\mathrm{e}}=1.224270 \times 10^{6}, p<.0001\right]$. The three-way interaction between target/foil, study size, and test size was significant in the error rate analysis $[F(1,23)=9.71$, $\left.M S_{\mathrm{e}}=1.0384 \times 10^{2}, p<.005\right]$ and marginal in the RT analysis $\left[F(1,23)=3.40, M S_{\mathrm{c}}=3.152304 \times 10^{5}, p<\right.$ .08]. These results reflect the fact that the study size $X$ test size interaction occurs for target trials but not for foils.

\section{Discussion}

The minor changes to Jolicoeur's (1987) method did not change the results significantly. Here too, shapes that 
were shown at the same size at the time of encoding and testing were recognized more quickly and accurately than were those shown at different sizes. This effect was not observed for the foils, but only for target trials, where the test shape appeared at one of two sizes in the study phase.

The different results for targets and foils can be explained easily. When a foil appeared on the screen, it may have been necessary to perform a search of all large and small shapes in memory to conclude that no such shape was presented at either size in the study session. This would lead to longer RTs for foils than for targets, and it would lead to RTs for foils being independent of test size (as well as, of course, the dummy-variable study size). However, on $50 \%$ of the occasions when a target appeared on the screen, it appeared at the same size as it did when studied. If indeed shapes are stored together with their size in memory, and if a target shape of incongruent size needs to be transformed in some way in order to match its internal representation, then we would expect that RT to recognize a target would be quicker if the test size corresponded to the study size. Thus, it is only RT for targets that would be expected to depend on the correspondence between study size and test size.

In contrast to Experiment 1, the subjects in Experiments 2 and 3 were asked to study shapes shown at only one size. Knowing the size at which all of the shapes were encoded may provide subjects with a shorter route to a correct rejection. For example, if a subject who studied only small shapes is presented with a large test shape, and if that subject realizes that an immediate size transformation is the most expedient route to a decision, then foils tested large will differ from foils tested small by exactly the amount of time necessary for that transformation. Thus, with a unique study size, there is reason to expect a test-size effect for both targets and foils. Indeed, in both Experiments 2 and 3, the foils exhibited a test-size effect in the anticipated direction. As was mentioned earlier, however, the more pertinent analyses are those of the target data. For this reason, the results for foils will no longer be presented in detail. ${ }^{3}$

\section{EXPERIMENT 2}

As was suggested in the introduction, when subjects are situated the same distance from the viewing screen during encoding and testing, retinal size and perceived size are confounded with one another. It was the purpose of Experiment 2, therefore, to tease apart retinal and perceived size by manipulating the distance from the subject to the monitor.

One group of subjects studied shapes at a small test size from a close test distance and performed a recognition test with shapes both at the same objective size as the study phase and at twice the objective size of the study phase. The decoupling of retinal size from perceived size was accomplished by having these subjects perform a portion of their test phase from the same distance as the study phase and a portion from twice the distance of the study phase. Note that from twice the study distance, size con- stancy dictates that the small shapes should be perceived as being the same size as in the study phase, despite the fact that the retinal image of such shapes is now half the size of the study phase. Similarly, large test shapes should be perceived as having a different size despite the identical sizes of the retinal images across the study and test phases.

The other group of subjects studied shapes at a large size from a far distance and performed a recognition test with shapes at both large and small objective sizes. Again, the subjects performed a portion of recognition trials from the same distance as the study phase and a portion from a distance that dissociated retinal size from perceived size (in this case, half the study distance).

If it is retinal size that is encoded as part of the representation of shape, then shapes studied and tested at the same retinal size should be recognized more quickly and/or accurately than shapes studied and tested at different retinal sizes. In terms of our design, to the extent that changing the test distance reverses the direction of the test-size difference, the retinal-size hypothesis is supported.

\section{Method}

Subjects. Forty-seven undergraduates and 1 graduate student at the University of Waterloo comprised the two groups of 24 subjects, who were paid for participation in this experiment. All subjects had normal or corrected-to-normal vision and were naive as to the purpose of the study.

Materials. The stimuli and apparatus were the same as those used in Experiment 1.

Procedure. Much of the procedure of Experiment 2 was identical to that of Experiment 1. Details that differ are mentioned below.

Two groups of subjects participated in a study phase and a test phase, as in Experiment 1. However, study size was no longer manipulated within subjects. Each group studied shapes at one size only and then was tested with shapes of two different sizes.

Study small/close group. The subjects studied small shapes from a distance of $66 \mathrm{~cm}$. The shapes subtended an average visual angle of $4.0^{\circ}$ along the longer of the horizontal or vertical axis. Following the study session, half of the subjects completed 20 recognition trials from the same distance (congruent test distance condition) and then the final $\mathbf{2 0}$ recognition trials from twice the study distance $(132 \mathrm{~cm})$ (incongruent test distance condition). The other half of the subjects completed the first 20 recognition trials from $132 \mathrm{~cm}$ (the incongruent test distance) and the second 20 recognition trials from $66 \mathrm{~cm}$ (the congruent test distance). The test phase was briefly interrupted after the first 20 recognition trials while the experimenter moved the table (at which the subject sat) and the chinrest to the appropriate test distance.

In the congruent test distance condition, the 20 recognition trials were comprised of 10 targets and 10 foils. Five targets and 5 foils were presented at the same objective size as in the study phase, and 5 targets and 5 foils were presented at twice the objective size of the study phase. Because this condition was nearly identical to Experiment 1, it was expected that shapes tested small would be recognized more quickly and/or accurately than shapes tested large, since the small objective size matched that of the study phase.

In the incongruent test distance condition, the 20 recognition trials were comprised of 5 large and 5 small targets, and 5 large and 5 small foils. However, the distance from the subject to the computer monitor was twice that in the study phase. As a result, the large test shapes subtended an average visual angle of $4.0^{\circ}$. Note that this is exactly the visual angle subtended by the shapes when studied at a small objective size from half the distance. Thus, in the incongruent test distance condition, the retinal size of the objectively large test shapes was the same as the retinal size of the objectively small 
studied shapes. The small test shapes, in contrast, which subtended an average visual angle of $2.0^{\circ}$, did not match in retinal size with the shapes in the study phase, but they did match in objective size. If the size-congruency effect observed in Experiment 1 is attributable to the objects' sharing the same retinal size across the study and test phases, then this group of subjects should recognize objectively large test shapes more quickly and/or more accurately than the objectively small shapes in the incongruent test distance condition.

Across each group of 8 subjects, every shape appeared once in all possible combinations of target/foil, test distance, and test size. As in Experiment 1, groups of shapes that appeared together in a particular cell of the design for one subject were distributed among the remaining cells for subsequent subjects, so that any two shapes appeared together in the same cell a minimum number of times.

Study large/far group. The subjects studied large shapes from a distance of $132 \mathrm{~cm}$. Again, the studied shapes subtended an average visual angle of $4.0^{\circ}$ in the study phase. The test phase was identical to that for the small/close group, described above. Thus, in the congruent test distance condition $(132 \mathrm{~cm})$, the subjects were expected to show a pattern similar to that of Experiment 1. In the incongruent test distance condition, objective and retinal size were dissociated from one another. If retinal size congruency is responsible for the size-congruency effect observed in Experiment 1, then objectively small shapes should be recognized more quickly and/or accurately than objectively large shapes, since the retinal size of the small shapes $\left(4.0^{\circ}\right)$ from a close distance $(66 \mathrm{~cm})$ matches that of the objectively large studied shapes from twice the distance $(132 \mathrm{~cm})$.

\section{Results}

Before looking at the data, it would be helpful to consider what would be expected were the size effects due solely to changes in retinal size (retinal-size hypothesis) or solely to changes in perceived size (perceived-size hypothesis). First, consider that the principal variables involved in the analyses were study size/distance (between subjects), test distance (within subjects), and test size (within subjects). Note that when the test and study phases are performed from the same distance (congruent test distance condition), the retinal- and objective-size hypotheses both predict a study size/distance $\times$ test size interaction, as retinal and perceived size are confounded. This would merely replicate the standard size-congruency finding of Experiment 1. However, when the test and study phases are performed from different distances (incongruent test distance condition), retinal and perceived sizes are dissociated and the retinal- and perceived-size hypotheses make different predictions.

Ignoring potential main effects of test size and study size, Figure 3 displays the two predictions for the incongruent test distance condition. The hypothetical data in the top portion conform to the prediction of the perceivedsize hypothesis. Note that the pattern is identical to that predicted for the congruent test distance condition. In short, the perceived-size hypothesis predicts that test distance does not affect the study size $\times$ test size interaction. On the other hand, the data in the bottom portion conform to the prediction of the retinal-size hypothesis. Here, the direction of the interaction has changed completely. Shapes studied large are recognized more efficiently when tested small, since the small shapes in the test phase (the test distance being "close") are the same retinal size as when studied. Similarly, shapes studied small are recog-
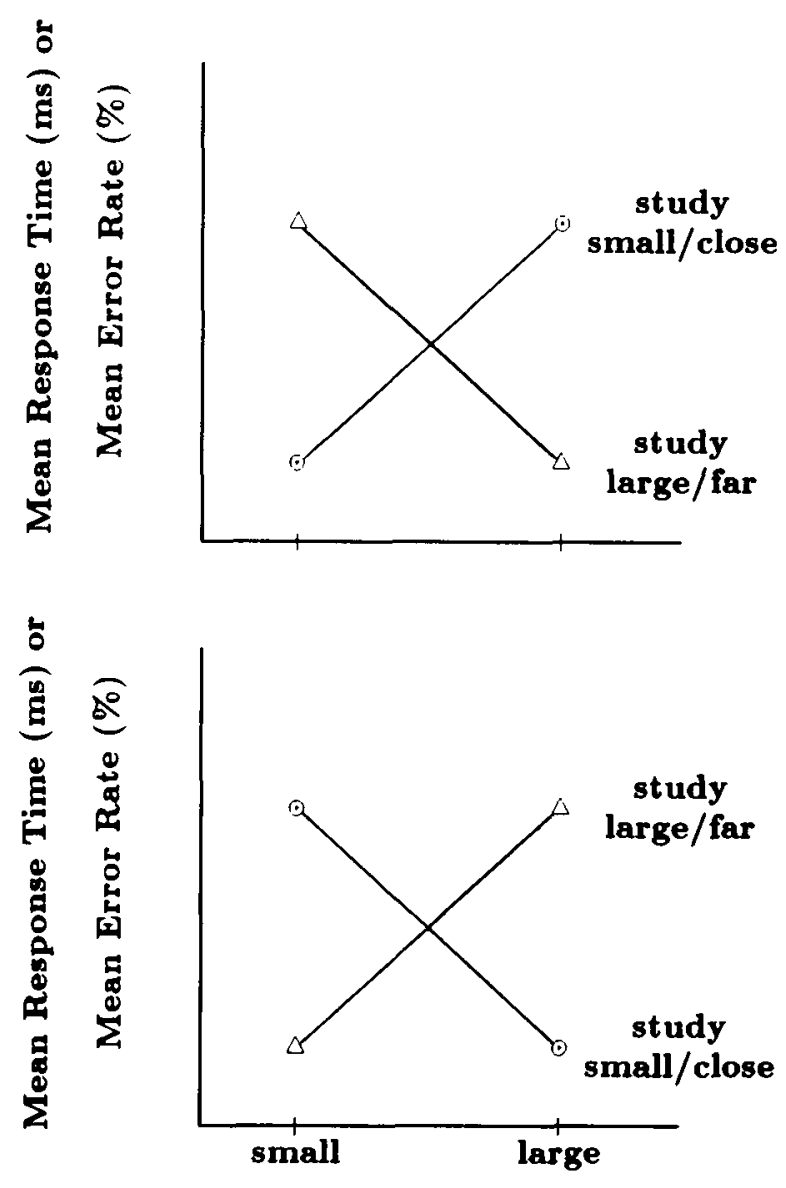

Test Size

Figure 3. Predictions conforming to the perceived-size hypothesis (top) and the retinal-size hypothesis (bottom) in the incongruent test distance condition of Experiment 2.

nized more efficiently when tested large, since the large shapes are the same retinal size as when studied (the test distance being "far"). The retinal-size hypothesis, then, predicts that changing the test distance will reverse the direction of the test-size effects that make up the study size by test size interaction, again resulting in a study size $x$ test size interaction, but in the opposite direction (see bottom of Figure 3).

Response times. The mean correct RTs and error rates on targets, for both groups of subjects (i.e., both study size/distances), both test distances, and both test sizes are presented in Table 1.

Study small/close and large/far groups combined. The mean correct RTs for the two groups, two test distances, and two test sizes were submitted to a repeated measures ANOVA that treated groups as a between-subject factor and test distance and test size as within-subject factors.

None of the statistical tests involving test distance were near significance (all $p s>.24$ ). Most importantly, there was no hint of a test distance $\times$ test size interaction $(F<1)$. Figure 4 displays the group $\times$ test size means, collapsed across test distance. The ANOVA performed on the collapsed data revealed a significant group $\times$ test 
Table 1

Mean Correct Response Times (RTs; in Milliseconds) and Mean Error Rates

(ERs; in Percent) for the Study Close/Small and Study Far/Large Groups at Each Test Distance for Each Test Size in Experiment 2

\begin{tabular}{|c|c|c|c|c|c|c|c|c|}
\hline & \multicolumn{4}{|c|}{ Study Small/Close Group } & \multicolumn{4}{|c|}{ Study Large/Far Group } \\
\hline & \multicolumn{2}{|c|}{ Test Close } & \multicolumn{2}{|c|}{ Test Far } & \multicolumn{2}{|c|}{ Test Close } & \multicolumn{2}{|c|}{ Test Far } \\
\hline & RT & ER & RT & ER & RT & ER & RT & ER \\
\hline & 1,192 & $\begin{array}{r}7.5 \\
75\end{array}$ & 1,235 & 11.7 & 1,109 & $\begin{array}{r}15.8 \\
0 ?\end{array}$ & 1,194 & 12.5 \\
\hline Test Large & 1,447 & 17.5 & 1,555 & 18.3 & 1,097 & 9.2 & 1,089 & 12.5 \\
\hline
\end{tabular}

Note-Close distance $=66 \mathrm{~cm}$; far distance $=132 \mathrm{~cm}$; small size $=2^{\circ}$ from far, $4^{\circ}$ from close; large size $=4^{\circ}$ from far, $2^{\circ}$ from close .

size interaction $\left[F(1,46)=15.89, M S_{\mathrm{e}}=9.038099 \times 10^{4}\right.$, $p<.0003$ ]. Collapsed across test distance, then, both groups tended to recognize shapes more quickly when they were the same perceived size across the study and test phases. That is, the difference between the RT means for shapes tested small and shapes tested large changes sign across the two groups $(-287 \mathrm{msec}$, for the small/close group; $+60 \mathrm{msec}$, for the large/far group). However, the test-size effect was not equally large for the two groups. This is reflected in the significant main effect of test size $\left[F(1,46)=6.94, M S_{e}=9.038099 \times 10^{4}, p<.02\right]$. Small shapes were recognized more quickly $(1,183 \mathrm{msec})$ on the
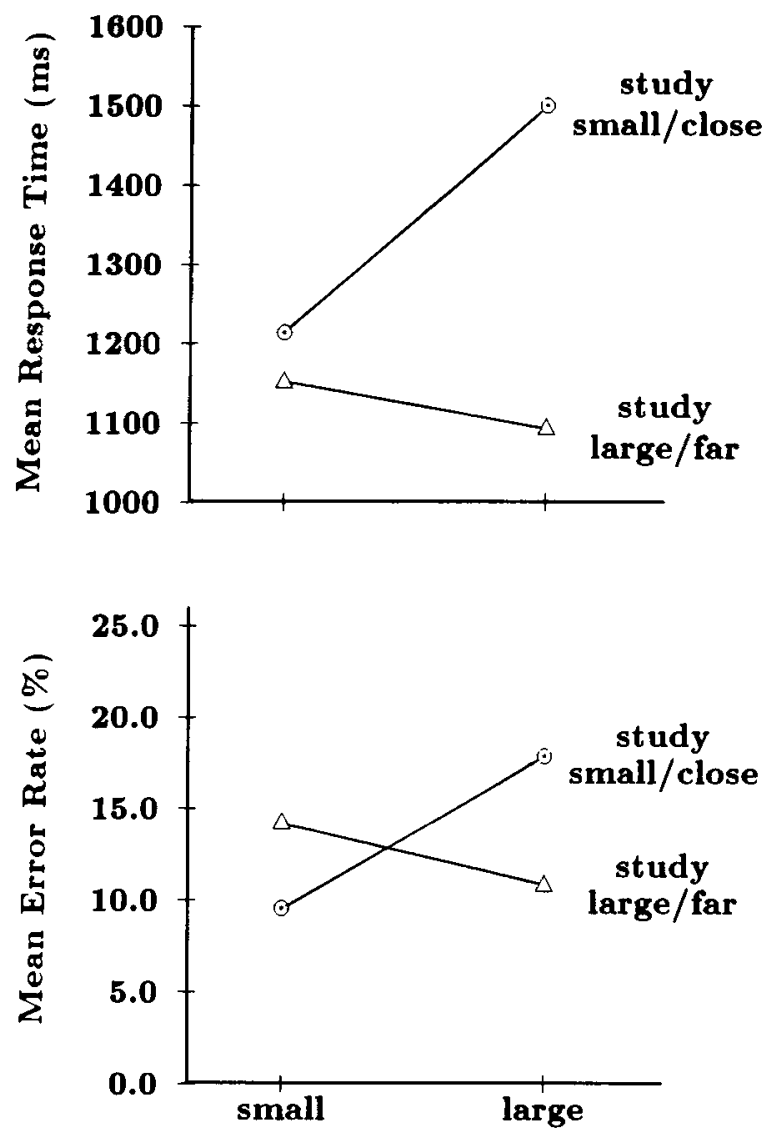

Test Size

Figure 4. Mean response times and mean percent error rates for targets at each test size for both groups in Experiment 2. whole than were large shapes $(1,296 \mathrm{msec})$. Also, the large/far group responded marginally faster $(1,122 \mathrm{msec})$ than did the small/close group $(1,357 \mathrm{msec})[F(1,46)=$ $\left.3.91, M S_{\mathrm{e}}=6.795545 \times 10^{3}, p<.06\right]$.

To more easily compare the results obtained with those predicted by the retinal- and perceived-size hypotheses, the data were separated by congruency of the test distance with the study distance. Mean RTs for the subjects from both groups were submitted to two repeated measures ANOVAs that treated study size/distance as a betweensubject factor and test size as a within-subject factor.

Congruent test distance. The study size/distance $\times$ test size means for the congruent test distance condition are displayed in the upper portion of Figure 5. As expected, study size/distance interacted significantly with test size $\left[F(1,46)=11.38, M S_{\mathrm{e}}=6.815789 \times 10^{4}, p<.01\right]$. The subjects who studied small shapes (from close) recognized those shapes more quickly when they were also small in the test phase. Similarly, the subjects who studied large shapes (from far) recognized those shapes more quickly when they were also large in the test phase.

Incongruent test distance. The study size/distance $x$ test size means for the incongruent test distance condition are displayed in the upper portion of Figure 6. Again, the interaction between study size/distance and test size was significant $\left[F(1,46)=7.17, M S_{\mathrm{e}}=9.222631 \times 10^{4}\right.$, $p<.02]$. As was true in the congruent test distance condition, the subjects recognized objects of congruent perceived size faster than they recognized objects of congruent retinal size when testing was done from a distance that dissociated the two.

The data were also separated by group and analyzed in repeated measures ANOVAs that treated test distance and test size as within-subject factors.

Study small/close group. There was a main effect of test size $\left[F(1,23)=21.64, M S_{\mathrm{e}}=9.156218 \times 10^{4}, p<\right.$ $.0002]$, with no hint of a test size $\times$ test distance interaction $(F<1)$. On the whole, small shapes were recognized much more quickly than were large shapes. Analysis of the simple main effects of test size revealed a significant difference both at the close test distance $(1,192$ vs. 1,447 msec) $\left[F(1,23)=12.35, M S_{\mathrm{e}}=6.304433 \times 10^{4}, p<\right.$ .002 ] and at the far test distance $(1,235 \mathrm{vs} .1,555 \mathrm{msec})$ $\left[F(1,23)=9.19, M S_{\mathrm{e}}=1.336361 \times 10^{3}, p<.006\right]$. In both cases, small shapes were recognized more quickly.

Study large/far group. No significant effects were found in this analysis. However, it is important to note that the 

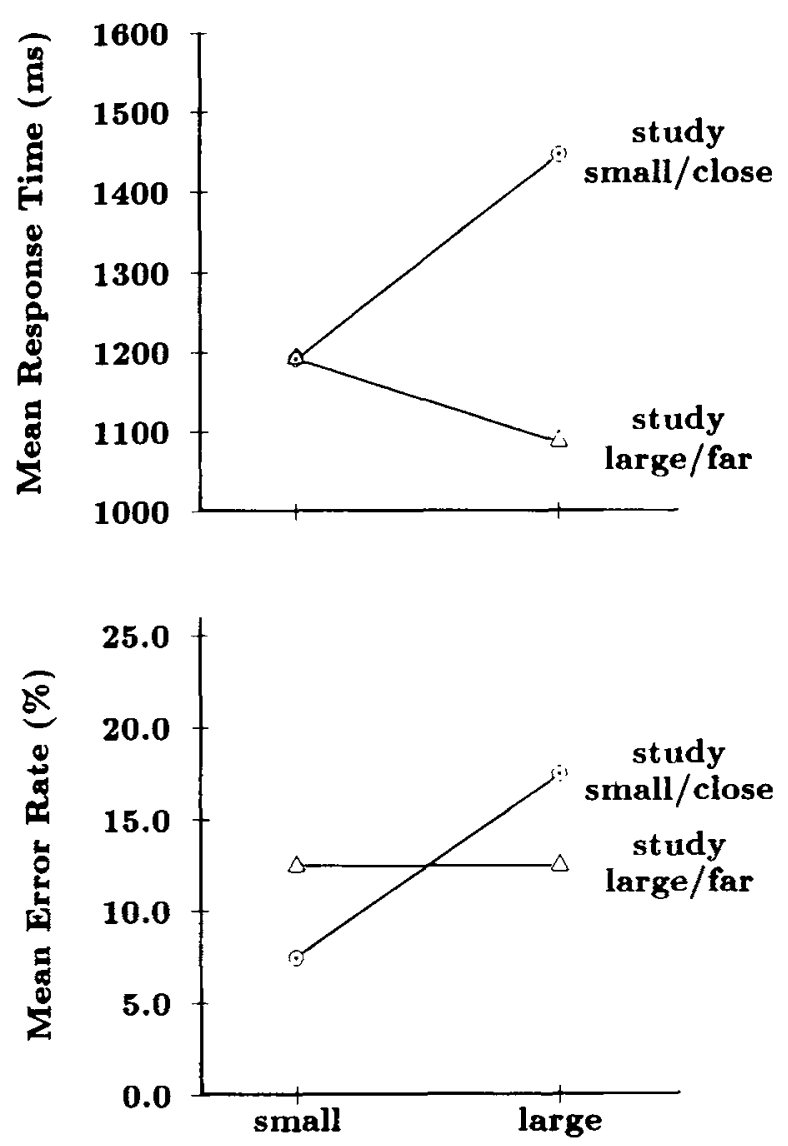

Test Size

Figure 5. Mean response times and mean percent error rates for targets at the congruent test distance in Experiment 2.

direction of the RT difference between items tested large and items tested small is consistent across the test distances and that this direction is the same as that which would be predicted by an influence of perceived size.

Error rates. The mean percent error rates on target trials for the two groups (study size/distances), two test distances, and two test sizes were also submitted to a repeated measures ANOVA that treated groups as a betweensubject factor and test distance and test size as withinsubject factors.

Study small/close and large/far groups combined. Again, none of the statistical tests involving test distance were near significance (all $p s>.25$ ), and there was no hint of a test distance $\times$ test size interaction $(F<1)$. The only significant effect in this analysis was the groups $\times$ test size interaction $\left[F(1,23)=6.79, M S_{\mathrm{e}}=2.406568 \times 10^{2}\right.$, $p<.02]$. The study small/close group subjects recognized small shapes more accurately, whereas the study large/far group subjects recognized large shapes more accurately (see bottom of Figure 4).

The error rates from the congruent and incongruent test distance conditions were submitted to the same analyses as were the corresponding RTs.
Congruent test distance. Although there were no significant effects in this analysis, the pattern of error rates making up the group $\times$ test size interaction did not suggest a speed-accuracy tradeoff (see bottom of Figure 5).

Incongruent test distance. Group interacted significantly with test size $\left[F(1,46)=4.47, M S_{\mathrm{e}}=2.376812 \times 10^{2}\right.$, $p<.04]$; small shapes were recognized more accurately in the study small/close group, and large shapes were recognized more accurately in the study large/far group (see bottom of Figure 6). As was true for the RTs, the subjects showed better memory for shapes that were the same perceived size in the study and test phases. No other effects approached significance (all $F \mathrm{~s}<1$ ).

Error rates were also separated by group and analyzed in repeated measures ANOVAs corresponding to those of the RTs.

Study small/close group. The only significant effect was that of test size $\left[F(1,23)=4.83, M S_{\mathrm{e}}=3.449041 \times 10^{2}\right.$, $p<.04$ ]. More errors were made on large shapes (17.9\%) than on small shapes $(9.6 \%)$. An analysis of the simple main effects revealed that, although the direction of the difference was the same at both test distances, small shapes were significantly more accurately recognized than were
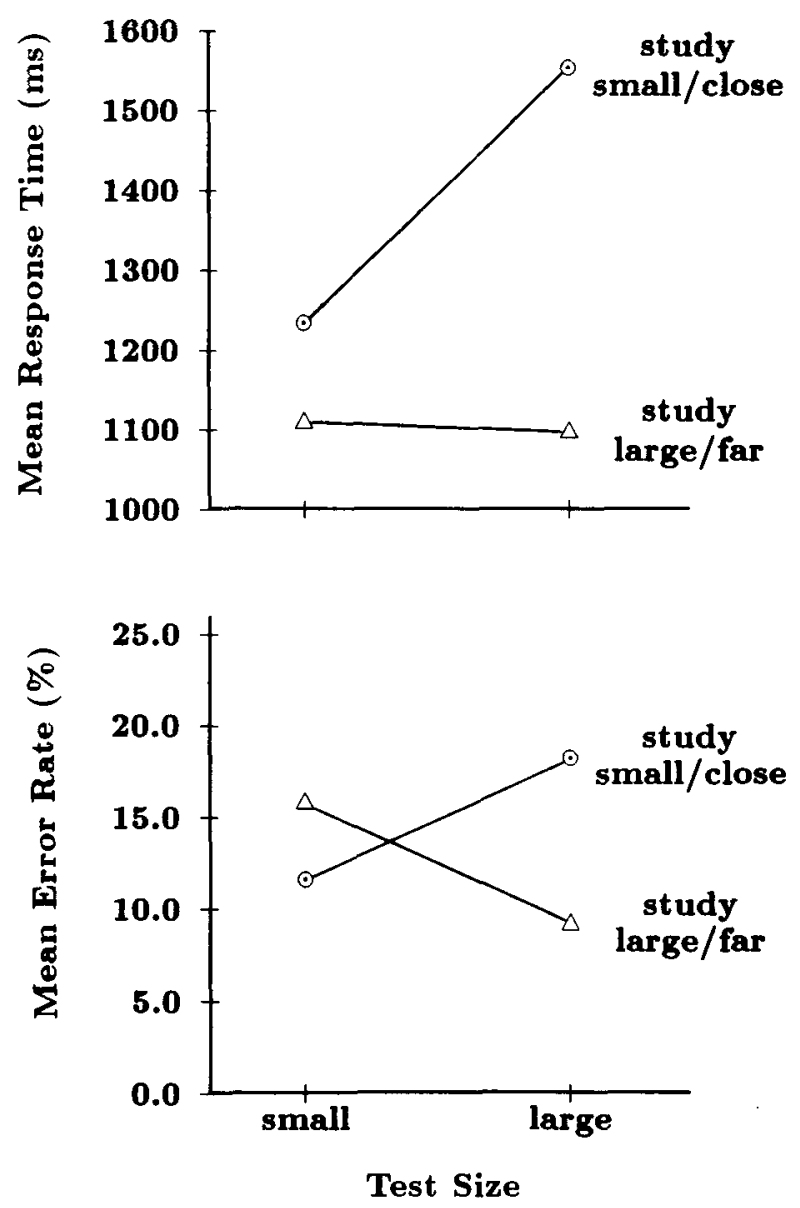

Figure 6. Mean response times and mean percent error rates for targets at the incongruent test distance in Experiment 2. 
large shapes only at the close test distance $(7.5 \%$ vs. $17.5 \%)$ $\left[F(1,23)=4.31, M S_{\mathrm{e}}=2.782193 \times 10^{2}, p<.05\right]$.

Study large/far group. Although there were no significant effects in the overall analysis, it should be noted that at the close test distance (the subjects studied from far), the subjects made more errors on the small shapes $(15.8 \%)$ than on the large shapes $(9.2 \%)$. In the analysis of simple main effects, this difference proved to be significant $\left[F(1,23)=4.60, M S_{\mathrm{e}}=1.159834 \times 10^{2}, p<.05\right]$. In other words, shapes of congruent perceived size were recognized more accurately than were shapes of congruent retinal size.

\section{Discussion}

The perceived-size hypothesis predicts that study size/ distance should interact with test size in the same manner in both the congruent and the incongruent test distance conditions. This is indeed what occurred in Experiment 2. In other words, when small shapes were studied from close, recognition speed and accuracy were better for small shapes at the far test distance (where the large shapes were the same retinal size). Furthermore, when large shapes were studied from far, recognition speed and accuracy were better for large shapes at the close test distance (where the small shapes were the same retinal size). The retinal-size hypothesis, which predicted that the direction of the test-size effects would be opposite in the congruent and incongruent test distance conditions, was obviously not substantiated.

The significant main effect of test size on RT replicated the effect that was found in Experiment 1. Therefore, it seems unlikely that small shapes were recognized more quickly than large shapes in the present experiments only by chance. If size scaling (see Besner, 1983; Besner \& Coltheart, 1976; Bundesen \& Larsen, 1975; Jolicoeur \& Besner, 1987; Larsen, 1985; Larsen \& Bundesen, 1978; Posner \& Mitchell, 1967; Sekuler \& Nash, 1972) is a simple analog transformation, then we might expect that the time needed to scale a large representation to a small size would be the same as that needed to scale a small representation to a large size. If so, one would expect no main effect of test size. The significant main effect of test size in Experiment 2 suggests either that scaling time depends on the direction of scaling (small to large or large to small) or that the absolute size of shapes may have processing consequences in our task as well.

Accommodating an effect of absolute size is not a simple matter, however, because it is not always the smaller shapes that are recognized faster. Jolicoeur (1987) described seven study size $\times$ test size interactions using RT data gathered using various stimuli, and under varying display and size ratio conditions. On some occasions, shapes tested small were recognized faster than shapes tested large; on others, the opposite was true. In similar studies by Kolers et al. (1985), this inconsistency appears in two, nearidentical, face-recognition studies. In the first, faces tested large and studied small were recognized more often than were faces tested small and studied large. In the second, the opposite was true. Kolers et al. (1985) suggested that perhaps different kinds of shapes have different ideal presentation sizes. Their proposal suggests a more general solution to the problem. If there is a stage of processing that precedes size normalization, and if that stage of processing is sensitive to absolute size, then nonsymmetric effects of test size would not rule out analog transformations. For example, the test-size effect in Experiment 2 can be accounted for by a normalization routine that is initiated later in time when a large shape appears than when a small shape appears. Why it would be initiated later for the large shapes used in the present experiments but for small shapes in other experiments is not clear at this time. In any case, main effects of test size do not prevent us from profitably studying issues of size ratio reflected by interactions between study size and test size.

A methodological issue not yet considered is that when the objective size of a shape is doubled, that shape takes up a larger proportion of the area of the computer monitor. As a result, the perceived-and retinal-size manipulation in Experiment 2 was confounded with the closeness of the shapes to the edge of the display monitor. Although we cannot rule out the possibility that size with respect to the monitor contributes to the size-congruency effect, Jolicoeur (1987) found a size-congruency effect in experiments where the size of the large shapes ranged from $30^{\circ}$ to $2.6^{\circ}$. It seems unlikely, therefore, that the size of shapes with respect to the monitor plays a predominant role in the size-congruency effect.

\section{EXPERIMENT 3}

The results of Experiment 2 show that shapes encoded at a particular size are recognized better when they are perceived as having the same size at the time of testing. Presumably, this occurs because the perceived size of a shape is stored as part of the representation of that shape in memory. If the perceived size of a test shape matches that of its corresponding internal representation, recognition is relatively quick, since there is no need to preempt the match by a scaling operation. Perceived size, for its part, is traditionally thought of as being determined jointly by the size of the retinal image of the object and the perceived distance of the observer from the object. The sizedistance invariance hypothesis (Epstein, Park, \& Casey, 1961; Kilpatrick \& Ittelson, 1953) is a formal statement of the geometric rule that describes the relationship between size and distance perception: "A retinal projection or visual angle of given size determines a unique ratio of apparent size to apparent distance" (Kilpatrick \& Ittelson, 1953 , p. 224).

Clearly, we assumed the size-distance invariance hypothesis to be true when we concluded that shapes displayed at the same objective size in Experiment 2 were perceived as being the same size, regardless of the test distance. Note, however, that since perceived size depends on perceived distance, the perceived-size-congruency effect leads to an advantage for objectively same-sized shapes in Experiment 2 only because relative distance was perceived veridically in both the study phase and the in- 
congruent test distance condition of the test phase. In other words, if subjects had ample depth cues to help them perceive distance in, say the study phase, but reduced depth cues leading to nonveridical distance perception in the test phase, then objectively same-sized shapes would no longer be the same perceived size across the study and test phases. Furthermore, assuming that congruency in perceived size leads to the size-congruency effect, the advantage in recognition for objectively same-sized shapes documented in Experiment 2 would be compromised.

In Experiment 3, subjects in one of two groups studied small shapes from $66 \mathrm{~cm}$ and then were tested on small and large shapes from $132 \mathrm{~cm}$ under reduced-viewing conditions (reduced group). A second group of subjects participated in the same task, but under full-viewing conditions ( full group). Obviously, the group tested under fullviewing conditions is expected to recognize objectively small shapes more quickly and accurately than large shapes, replicating the perceived-size-congruency effect found in Experiments 1 and 2. To properly anticipate the results of the group tested under reduced-viewing conditions, a brief consideration of previous studies of size and distance perception under reduced-viewing conditions is warranted.

In their pioneering work in the field, Holway and Boring (1941) had subjects adjust the size of a comparison shape to that of a standard shape whose distance from the subject varied. Subjects viewed the shapes monocularly, through an artificial pupil, and down a long reduction tube. They found that, under these conditions, subjects adjusted the comparison stimulus to be very nearly the same retinal size as the standard stimulus (see also Lichten \& Lurie, 1950). Holway and Boring's (1941) interpretation of their data was that, under reduced conditions, perceived size is based directly on retinal size rather than indirectly on distance. This interpretation subsequently received considerable support (Brosgole, PlaHovinsak, Roig, \& Notaro, 1985; Epstein \& Landauer, 1969; Landauer \& Epstein, 1969).

However, Gogel (1965, 1969a, 1969b, 1971; Gogel \& Da Silva, 1987a, 1987b) has convincingly argued that subjects do not directly perceive retinal size. Instead, in the absence of distance cues, subjects assume a particular distance and make size estimates accordingly. The tendency to adopt a particular distance in the absence of distance cues, then, implies that Holway and Boring's (1941) retinal-size matches were a result of implied equidistance of the standard and comparison shapes (Gogel, 1965, 1969a; see also Wallach \& McKenna, 1960). More generally, the equidistance tendency states that as depth cues between two visible objects are reduced, the two objects appear increasingly near each other in depth, regardless of their physical depth separation (Gogel, 1965, 1969a).

At any rate, if Gogel's $(1965,1969 a, 1969 b, 1971)$ view that perceived size is a function of perceived distance under reduced-viewing conditions is correct, then we can anticipate the results of Experiment 3, contingent upon subjects' perception of distance under reduced-viewing conditions. If subjects in the reduced group perceive their test distance to be less than $132 \mathrm{~cm}$, then the size- congruency effect should be attenuated in the reduced group. This follows because the underestimated test distance should render the perceived size of the small test shapes to be smaller than their perceived size at the time of study. Similarly, to the extent that subjects perceive themselves to be closer to $66 \mathrm{~cm}$ than to the actual $132 \mathrm{~cm}$ from the computer monitor, the perceived size of the large test shapes should approach the perceived size of the small studied shapes. Only in the event that perceived distance is greater than $132 \mathrm{~cm}$ should we expect the size-congruency effect to be enhanced in the reduced group relative to that in the full group.

\section{Method}

Subjects. Forty-eight undergraduate students at the University of Waterloo were paid for their participation in the experiment. The subjects were assigned to one of two groups on the basis of the order of their participation. The data for 7 additional subjects were excluded owing to near-chance performance. All subjects had normal or corrected-to-normal vision and were unaware of the purpose of the experiment prior to participation.

Materials. The shapes used in Experiment 3 were the same as those used in Experiments 1 and 2. The small shapes subtended visual angles of $4^{\circ}$ from $66 \mathrm{~cm}$ and $2^{\circ}$ from $132 \mathrm{~cm}$, whereas the large shapes subtended visual angles of $8^{\circ}$ from $66 \mathrm{~cm}$ and $4^{\circ}$ from $132 \mathrm{~cm}$.

Procedure. Any details of the procedure not mentioned here can be assumed to have been identical to those of Experiment 1 .

The study phase was identical for the two groups; each subject studied 20 small shapes from a distance of $66 \mathrm{~cm}$. The test phase differed for the two groups in that one group of subjects (full group) performed the recognition test with full-distance cues and the other group of subjects (reduced group) performed the recognition test with reduced-distance cues.

Both groups of subjects performed the recognition test from a distance of $132 \mathrm{~cm}$. During the test phase, half of both the target and foil shapes were shown large and half were shown small. Across each block of 8 subjects, each shape was represented once in each of the cells created by the factorial combination of the two groups, two test sizes, and target/foil conditions. The order of presentation of the shapes within both the study phase and the test phase was random. No more than four consecutive targets or foils were allowed to appear in the test phase.

For both the full group and the reduced group, the study and test phases took place in adjacent rooms using identical Amiga microcomputers. Different rooms were used so that the apparatus needed to reduce distance cues could be set up in advance of the subjects' arrival and left intact between subjects in the reduced group. Eight subjects from the full group were run initially, then 16 from the reduced group, 16 from the full group, and finally 8 more from the reduced group. Such an ordering reduced to a reasonable amount the setting up and dismantling of equipment while controlling for possible effects of testing order across the two groups.

The subjects in the full group sat in a fully lit room, with an unobstructed view of the monitor in front of them. The subject rested his/her chin in a chinrest and placed the index finger of each hand on the response buttons on the table in front of him/her. The subjects were asked to respond as quickly and accurately as possible by pressing the dominant-hand response button for shapes that they had studied and the nondominant-hand response button for shapes they had not studied. Furthermore, each subject was given a detailed explanation of what a speed-accuracy tradeoff was; they were instructed to avoid engaging in such a tradeoff while responding as quickly as possible. The detailed instructions were given to the subjects in order to try to reduce the number of different ways in which the response instructions would be interpreted. Previous 
studies had demonstrated that subjects vary considerably in the importance they attach to the speed, as opposed to the accuracy, part of the instructions.

Each subject in the reduced group sat in a dark room, with an eye patch over the nondominant eye, and looked through a reduction tube whose end was flush with the computer monitor. The reduction tube had a rectangular plastic frame within which was a cylindrical tube made of black bristol board. The end closest to the subject was covered by another piece of bristol board in which two eye holes were cut. Small paper cylinders, $100 \mathrm{~mm}$ in length and the same diameter $(17 \mathrm{~mm})$ as the eye holes, were lined with black paper and placed in the eye holes such that only a minuscule portion $(2 \mathrm{~mm})$ protruded back toward the subject. Inside the reduction tube, the far end of the small cylinders rested on a cardboard platform lined with tape to keep the cylinders in place. The intent of the eye patch, of course, was to have the subject view the shapes monocularly. The reduction tube obscured the subject's view of the environment around the computer monitor. The small cylinders within the eye holes made it very difficult for the subject to move the head without losing sight of the monitor, thus reducing the possibility that the subject could pick up movement parallax cues to depth. Finally, a large sheet of cardboard was hung from the ceiling, with an area cut out to fit tightly around the reduction tube. The cardboard obstructed the subject's view of approximately three fourths of the testing room. Again, the subjects were instructed in detail to respond as quickly and accurately as possible by pressing the dominant-hand response button for shapes they had studied and the nondominant-hand response button for shapes not studied.

Following the test session, the subjects in the reduced group were asked to compare both their distance from the monitor in the test phase and the size of the shapes in the test phase with those in the study phase. The subjects were asked if the distance between themselves and the monitor was the same in the two phases, greater in the test session, or smaller in the test session. They were then asked whether the size of the shapes in the study phase, relative to the small and large shapes in the test phase, was closer to the small test size, closer to the large test size, or midway between the two test sizes.

\section{Results}

Distance and size comparisons. All 24 subjects in the reduced group judged that they were further from the screen in the test phase than in the study phase. More than half of these subjects mentioned that the reduction apparatus gave them the impression that they were looking down a long tunnel. Thirteen subjects said that the size of shapes during the study phase was closest to the large test size, 7 said it was closest to the small test size, and 4 said it was in between.

Note that we can infer from the relative distance judgments that the subjects uniformly believed themselves to be farther than $66 \mathrm{~cm}$ from the screen in the test phase. Moreover, from the relative size judgments, we may indirectly infer that the majority of the subjects made their size estimates on the basis of a perceived distance that was closer than the actual $132-\mathrm{cm}$ distance to the screen (objects that appear closer than they actually are also appear smaller). Further evidence that perceived distance was less than $132 \mathrm{~cm}$ comes from the subjects' ability to see the tunnel-like form of the small reduction tube, meaning that there was enough light emitted from the monitor to illuminate the inside of the reduction tube. This light may have formed a visible aperture at the far edges of the tube, not far from the observer. With the aperture be- ing spatially localized, the equidistance tendency (Gogel, 1965,1969 a) may have caused the stimuli to appear close to the same distance as the aperture and, thus, closer to the observer than the actual $132 \mathrm{~cm}^{4}$

As a result, the objectively small shapes in the test phase for the reduced group were not the same perceived size as the shapes of the study phase. Instead, the perceived size of the studied shapes must have been somewhere in between that of the small and large test shapes. This leads to the prediction that the size-congruency result expected in the full group should be attenuated in the reduced group.

Response times. The mean correct RTs on target trials for each subject were submitted to repeated measures ANOVAs for each group individually, treating test size as the lone within-subject variable. The data for both groups were submitted to a third repeated measures ANOVA, which treated groups as a between-subject factor.

The mean correct RTs to targets for each group at each test size are presented in the top portion of Figure 7. The subjects in the full group recognized small shapes significantly faster than large shapes $(1,054 \mathrm{vs} .1,161 \mathrm{msec})$ $\left[F(1,23)=4.61, M S_{\mathrm{e}}=2.997638 \times 10^{4}, p<.05\right]$. The subjects in the reduced group, however, showed a nonsignificant difference in the opposite direction $(1,333$ vs.
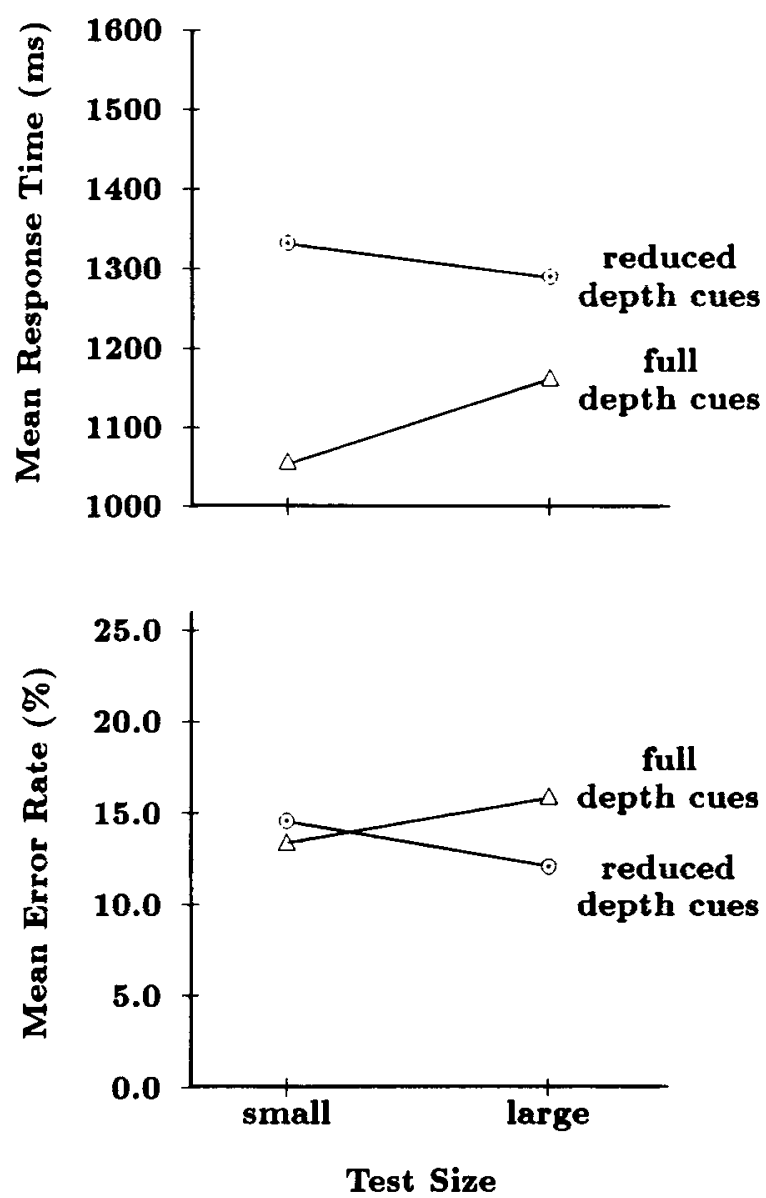

Figure 7. Mean response times and mean percent error rates for targets in Experiment 3. 
$1,291 \mathrm{msec})(F<1)$. In the combined groups analysis, this interaction between groups and test size was significant $\left[F(1,46)=3.56, M S_{\mathrm{e}}=3.762810 \times 10^{4}, p<.035\right.$; one-tailed.$^{5}$

Error rates. The mean percent error rates on target trials for each subject were submitted to analyses corresponding to those described for the RTs. The bottom portion of Figure 7 presents the test-size means for each group.

There were no significant effects in either the individual or the combined groups analyses. It should be noted, however, that the direction of the test-size effects for the full and reduced groups were the same as in the corresponding RT data (13.3\% vs. $15.8 \%$, in the full group; $14.6 \%$ vs. $12.1 \%$, in the reduced group). Therefore, a speedaccuracy tradeoff was not responsible for the pattern of RTs described above.

\section{Discussion}

Results from the subjects in the full group, on target trials, replicated the perceived-size-congruency effect found in Experiment 2. Shapes studied small were recognized more quickly when they were shown small in the test phase, despite the change in distance. The subjects in the reduced group recognized shapes equally quickly at both sizes, suggesting that the absence of distance cues impeded whatever it was that allowed for better recognition of small shapes in the full group. The most likely explanation is that these subjects did not perceive the shapes as having the same size either because they were unable to gauge distance accurately or because visual angle played a more prominent role in determining size.

The results of Experiment 3 also help to resolve a methodological issue that has been ignored to this point in time. Because the width of the constituent lines of the shapes was the same at the two sizes, it can be argued that the larger shapes had a slightly different texture, effective contrast, and absolute brightness than did the smaller shapes. That is, the pixel ratio of line to background was higher for small shapes than for large shapes. Therefore, shapes of identical perceived size in Experiment 2 also had identical line-to-background pixel ratios, while shapes of identical retinal size had different line-to-background pixel ratios. Congruency in perceived size, then, was confounded with congruency in line-to-background pixel ratio. In Experiment 3 , however, the pixel ratio of the small test shapes was congruent with that of the studied shapes for both groups, yet these shapes were better recognized only in the full group, where adequate cues to distance were available. Thus, the congruency of line-to-background pixel ratio can be presumed not to have played a predominant role in the results of Experiment 2.

This is not to say that the reduction conditions were completely successful in eliminating all depth cues. For example, the subjects were able to detect light reflecting off of the sides of the small reduction tube, giving them the impression that they were looking down a tunnel. Also, in that the width of the constituent lines of the shapes was constant, the subjects could have used this information, as well as accommodation cues, to determine that they were farther from the screen in the test phase than in the study phase. The point to be made, however, is that, even under imperfectly reduced conditions, veridical distance perception is unlikely. Our results show that indeed distance, and thus size, was perceived nonveridically under our reduced conditions, thereby leading to the attenuation of the size-congruency effect.

\section{GENERAL DISCUSSION}

Experiment 1 showed that the size-congruency effect in recognition memory is robust, by replicating the effect with a modified version of the methodology used originally by Jolicoeur (1987). The results of Experiments 2 and 3 converge in suggesting that the size-congruency effect in recognition memory is the result of perceived size being encoded as a functional part of the representation of shape.

We have based the conclusion that size is a part of longterm memory representations of shape on the assumption that recognition occurs through the matching of an external shape with a unique internal representation of that shape. However, it is possible that there are a number of different levels of representation for every shape in memory and that size information may be available at an intermediate level of representation instead of, or as well as, at a permanent, long-term level of representation. If size congruency with an intermediate level representation can facilitate memory, then the size-congruency effect need not imply that the more permanent representations of shape are size dependent. As a result, the present results should be considered in light of models of recognition that posit multiple levels of representation.

An example of a multistage model is that proposed by Marr and Nishihara (1978; Marr, 1982) in which stimulus equivalence is achieved by the transformation of vantage-point-specific representations (primal sketch, $21 / 2$ dimensional sketch) that are defined with respect to a viewer-centered coordinate frame, to vantage-pointindependent representations (3-dimensional model) that are defined within an object-centered coordinate frame. The intermediate, vantage-point-specific representations contain information about retinal size. The 3-dimensional models contain information about perceived size (retinal size corrected for viewer-centered distance). Both levels of representation could be involved in the recognition process, provided they are retained in memory and accessible during recognition. The present results, however, constrain which level of representation is apparently involved in the size-congruency effects explored in the present article. Our results suggest that perceived size is controlling the size-congruency effect. Because the first level of representation at which perceived size is represented explicitly is the level of 3-dimensional models, our results suggest that the primal sketch and $21 / 2$-dimensional sketch are not playing a functional role in the sizecongruency effect.

It should also be noted that insofar as a model deals with the various problems of changing vantage points 
(size, orientation, location) in the same manner, one might expect the effects of such variables on recognition to be similar in nature. For example, since we have found recognition memory not to depend on retinal size in the present experiments, one might also expect recognition memory not to depend on retinal orientation. Although there is some evidence to suggest that recognition of novel shapes is more sensitive to environmental than to retinal orientation (Rock \& Heimer, 1957), more recent work by McMullen and Jolicoeur (1990) suggests that if subjects are aware that shapes may be shown at different orientations, a retinally aligned frame of reference is likely to be adopted in recognition of disoriented shapes. Indeed, the subjects were aware that shapes could be presented at different sizes in the present experiments because they were instructed that shapes differing only in size were to be considered identical. This discrepancy between the reference frame used to compensate for changes in size and orientation in the recognition process may be cause for concern for models that group size and orientation together in an attempt to resolve, in a single step, the problems introduced by changing vantage points.

Another account of the size-congruency effect that obviates the need for size-dependent representations is one that assumes that encoding processes are sensitive to size, but that representations themselves do not represent size explicitly. For example, Goldmeier (1972) has noted that changing the size of a shape can affect the way in which an observer "groups" its elements. If a shape is encoded in a group-dependent format, but one where size is not directly represented, then size-dependent recognition may occur as a by-product of the different groupings. Other theories downplay the role of structural representations altogether and instead argue that memory consists of the procedures used to encode a stimulus (Kolers \& Roediger, 1984). As such, memory would be sensitive to an attribute of shape to the extent that the attribute has an impact on the way a stimulus is encoded. Although it is possible that such encoding processes are most affected by perceived size, changes in grouping, for instance, seem more naturally accounted for by changes in retinal size. The fact that the size-congruency effect depends on perceived size and not retinal size, therefore, makes an encoding account of the data less attractive than a representation account.

Various alternative explanations have been offered, but the most convincing conclusion based on past and present results remains that the perceived size of a shape is a functional part of its internal representation. Furthermore, the results presented in this article suggest that, although human memory may not be able to afford to have an entry for every vantage-point-dependent representation of each and every object, it seems able to afford more than one instance of a particular shape category when these instances typically differ in their perceived size. As such, the present results provide additional support for models of pattern recognition that make use of a number of specific representations for a shape, as well as transformations that mediate recognition when the input does not match exactly any one of the encoded representations (see Jolicoeur, 1985, 1988, 1990; Jolicoeur \& Milliken, 1989; Takano, 1989; Tarr \& Pinker, 1989).

In the case of pattern size, changes in vantage point are unlikely to result in different internal representations, since it seems that the mechanisms that mediate size constancy in perception also govern the nature of the memory representations of visual shape. Such a pattern of results is exactly what we would expect if there are strong functional relationships between what is perceived and what is remembered (see Kosslyn, 1980).

\section{REFERENCES}

BESNER, D. (1983). Visual pattern recognition: Size preprocessing reexamined. Quarterly Journal of Experimental Psychology, 35A, 209-216.

Besner, D., \& Colthenrt, M. (1976). Mental size scaling examined. Memory \& Cognition, 4, 525-531.

Brosgole, L., Plahovinsak, T. J., Roig, M., a Notaro, J. P. (1985). The role of perceived distance in determining apparent visual size. Bulletin of the Psychonomic Society, 23, 489-492.

BUNDESEN, C., LARSEN, A. (1975). Visual transformation of size. Journal of Experimental Psychology: Human Perception \& Performance, 1, 214-220.

Epstein, W., LANDAuer, A. A. (1969). Size and distance judgments under reduced conditions of viewing. Perception \& Psychophysics, 6, 269-272.

Epstein, W., Park, J., \& Casey, A. (1961). The current status of the size-distance invariance hypothesis. Psychological Bulletin, 58, 491-514.

GOGEL, W. C. (1965). Equidistance tendency and its consequences. Psychological Bulletin, 64, 153-163.

GOGEL, W. C. (1969a). Equidistance effects in visual fields. American Journal of Psychology, 82, 342-349.

GOGEL, W. C. (1969b). The sensing of retinal size. Vision, 9, 1079-1094.

GOGEL, W. C. (1971). The validity of the size-distance invariance hypothesis with cue reduction. Perception \& Psychophysics, 9, 92-94.

GoGEL, W. C., DA Silva, J. A. (1987a). Familiar size and the theory of off-sized perceptions. Perception \& Psychophysics, 41, 318-328.

GoGEL, W. C., \& DA Silva, J. A. (1987b). A two-process theory of the response to size and distance. Perception \& Psychophysics, 41, 220-238.

GoLDMEIER, E. (1972). Similarity in visually perceived forms. Psychological Issues, 8(1, Whole No. 29), 1-135.

Holway, A. H., Boring, E. G. (1941). Determinants of apparent visual size with distance variant. American Journal of Psychology, 54, 21-37.

JolicoeUR, P. (1985). The time to name disoriented natural objects. Memory \& Cognition, 13, 289-303.

Jolicoeur, P. (1987). A size-congruency effect in memory for visual shape. Memory \& Cognition, 15, 531-543.

JolicOEUR, P. (1988). Mental rotation and the identification of disoriented objects. Canadian Journal of Psychology, 42, 461-478.

Jolicoevr, P. (1990). On the role of mental rotation and feature extraction in the identification of disoriented objects: $A$ dual-systems theory. Mind \& Language, 5, 387-410.

Jolicoeur, P., Besner, D. (1987). Additivity and interaction between size ratio and response category in the comparison of sizediscrepant shapes. Joumal of Experimental Psychology: Human Perception \& Performance, 13, 478-487.

Jolicoeur, P., \& MILLIKEN, B. (1989). Identification of disoriented objects: Effects of context of prior presentation. Joumal of Experimental Psychology: Learning, Memory, \& Cognition, 15, 200-210.

KILPATRICK, F. P., ITTELSON, W. H. (1953). The size-distance invariance hypothesis. Psychological Review, 60, 223-231.

Kolers, P. A., DuchNicky, R. L., Sundstroem, G. (1985). Size in the visual processing of faces and words. Journal of Experimental Psychology: Human Perception \& Performance, 11, 726-751. 
Kolers, P. A., \& Rogdiger, H. L. (1984). Procedures of mind. Journal of Verbal Learning \& Verbal Behavior, 23, 425-449.

Kosslyn, S. M. (1980). Image and mind. Cambridge, MA: Harvard University Press.

Landauer, A. A., Epstein, W. (1969). Does retinal size have a unique correlate in perceived size? Perception \& Psychophysics, 6, 273-275.

LARSEN, A. (1985). Pattem matching: Effects of size ratio, angular difference in orientation, and familiarity. Perception \& Psychophysics, 38 , 63-68.

LARSEN, A., \& Bundesen, C. (1978). Size scaling in visual pattern recognition. Journal of Experimental Psychology: Human Perception \& Performance, 4, 1-20.

Lichiten, W., LuRIE, S. (1950). A new technique for the study of perceived size. American Journal of Psychology, 63, 281-282.

MARR, D. (1982). Vision. San Francisco: W. H. Freeman.

MARR, D., NishiHARA, H. K. (1978). Representation and recognition of the spatial organization of three-dimensional shapes. Proceedings of the Royal Society of London, Series B, 200, 269-294.

McMullen, P. A., Jolicoeur, P. (1990). The spatial frame of reference in object naming and discrimination of left-right reflections. Memory \& Cognition, 18, 99-115.

Posner, M. I., Mitchell, R. F. (1967). Chronometric analysis of classification. Psychological Review, 74, 392-409.

Rock, I. (1956). The orientation of forms on the retina and in the environment. American Journal of Psychology, 69, 513-528.

Rock, I. (1973). Orientation and form. New York: Academic Press.

Rock, I. (1974). The perception of disoriented figures. Sciensific American, 230, 78-85.

RoCk, 1., Di VITA, J., BARBeITo, R. (1981). The effect on form perception of change of orientation in the third dimension. Journal of Experimental Psychology: Human Perception \& Performance, 7 , 719-732.

Rock, 1., Heimer, W. (1957). The effects of retinal and phenomenal orientation on the perception of form. American Joumal of Psychology, 70, 493-511.

SEKULER, R., NASH, D. (1972). Speed of size scaling in human vision. Psychonomic Science, 27, 93-94.

TAKANO, Y. (1989). Perception of rotated forms: A theory of information types. Cognitive Psychology, 21, 1-59.

TANner, P. P., Jollcoeur, P., Cowan, W. B., Booth, K., Fishman, F. D. (1989). Antialiasing: A technique for smoothing jagged lines on a computer graphics image-An implementation for the AMIGA. Behavior Research Methods, Instruments, \& Computers, 21, 59-66.
TARr, M. J., Pinker, S. (1989). Similarity information versus relational information: Differences in the time course of retrieval. Cognitive Psychology, 21, 139-155.

WALLACH, H., MCKenNA, V. V. (1960). On size perception in the absence of cues for distance. American Joumal of Psychology, 73, 458-460.

\section{NOTES}

1. Antialiasing is a technique used to smooth the jagged edges that characterize straight lines drawn by simple line-drawing algorithms on many microcomputers.

2. Correct RTs were submitted to an outlier analysis that eliminated suspiciously large or small RTs $(3.3 \%, 5.8 \%$, and $3.1 \%$ of the data in Experiments 1, 2, and 3, respectively). For each cell in the design, the highest and lowest RTs were temporarily excluded and the mean and standard deviation of the remaining RTs were calculated. An upper limit of the mean plus four standard deviations and a lower limit of the mean minus four standand deviations were set. If the high temporarily excluded RT was less than the upper limit, then it was reincluded in the cell. Similarly, if the low excluded RT was greater than the lower limit, then it was reincluded in the cell. If either or both RTs were not reincluded, then the above procedure was repeated with the remaining members of the cell. The analysis ended when both temporarily excluded RTs were reincluded in the cell.

3. Note that extensive analyses of the data gathered on foil trials in all experiments were conducted. However, since the results in no way contradict our interpretation of the target data, and since signal detection measures are not applicable to reaction time data, we decided that their inclusion in the paper would result in unnecessary distraction.

4. Under completely reduced conditions, the specific distance tendency causes objects to be seen at a distance of between 2 and $3 \mathrm{~m}$ (Gogel, 1971). However, under partially reduced conditions, the equidistance tendency is the more powerful in determining perceived distance (Gogel, 1965,1969 ).

5. Our prediction based on the distance and size estimates of subjects was that the size-congruency effect found in the full group would be attenuated in the reduced group. This is in accord with both Gogel's $(1965,1969$ a) view of size and distance perception and with Holway and Boring's (1941) view that retinal size can be perceived directly.

(Manuscript received July 18, 1990; revision accepted for publication July 25,1991 .)

\section{Notices and Announcements}

\section{Call for Assistance in The Compilation of a History of the Psychonomic Society}

The Governing Board of the Psychonomic Society is pleased to announce that Robert C. Bolles has agreed to serve as the first Historian of the Society.

All members who might have information relevant to this undertaking are invited to send it directly to Dr. Bolles. Founding members and those who attended the early meetings are especially encouraged to record their reminiscences. While Dr. Bolles hopes to collect as much information as possible relevant to the history of the Society, he will concentrate first on the early history.

Dr. Bolles's address is Department of Psychology, University of Washington, Seattle, Washington 98195 (phone: 206-543-2631). 